\title{
The humble
}
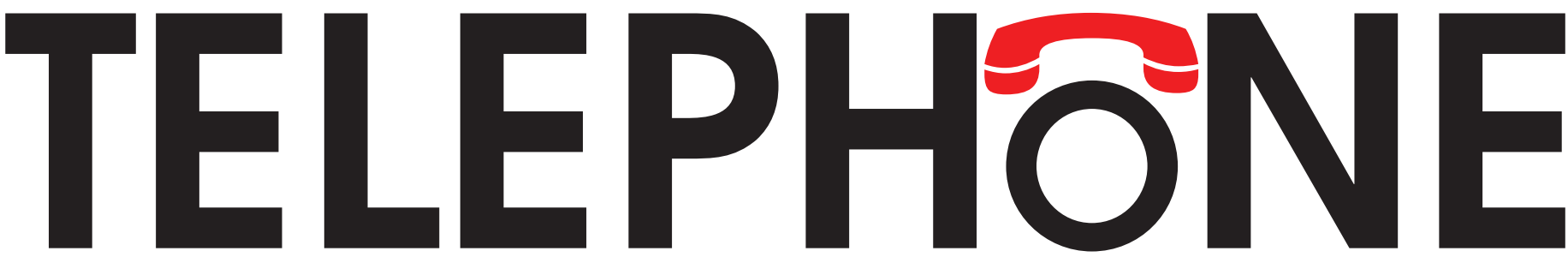

\section{Caelen King of RevaHealth.com* says that even in 2010, the telephone should be your dental practice's best friend.}

\section{W hatever the job you are given to perform, having the correct tools at your disposal is essential to completing it satisfactorily. You}

wouldn't dream of starting to treat a patient if you didn't have the right instruments to hand. Similarly, you wouldn't let someone perform a procedure if they hadn't had the correct training in advance.

The same logic should apply equally to administrative staff as to clinical staff. Administrative jobs are vital to the smooth running of your dental practice, and if

* Caelen King is founder and CEO of RevaHealth.com, an online search engine that helps over 3 million people a year to find and contact health clinics worldwide. Founded in 2006, it currently stores information on over 60,000 clinics in the UK and Ireland and thousands more in 50 other countries around the world. Visit the website at www. revahealth.com.

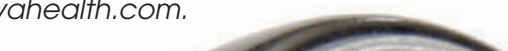

administrative staff are not making the best use of the tools available to them, profitability will suffer.

At RevaHealth.com we help dental practices connect with patients using the internet, so it may surprise you to hear that the tool we advise practices to use the most is the humble telephone. It really should be your practice's best friend.

\section{Weighing up the numbers}

Patients already decide to use either the internet or the phone to contact your dental practice, but have you ever looked at the numbers? Recent analysis of user behaviour on our own website shows that twice as many patients prefer to look up a practice's phone number rather than email them, and that is when they are already online.

In addition to this is the fact that there are 19.3 million internet connections in the UK compared to 33.2 million landlines and a staggering 77.4 million mobile phone accounts. Even taking into account that households share internet connections, the phone still far outweighs the internet as the communication tool of choice.

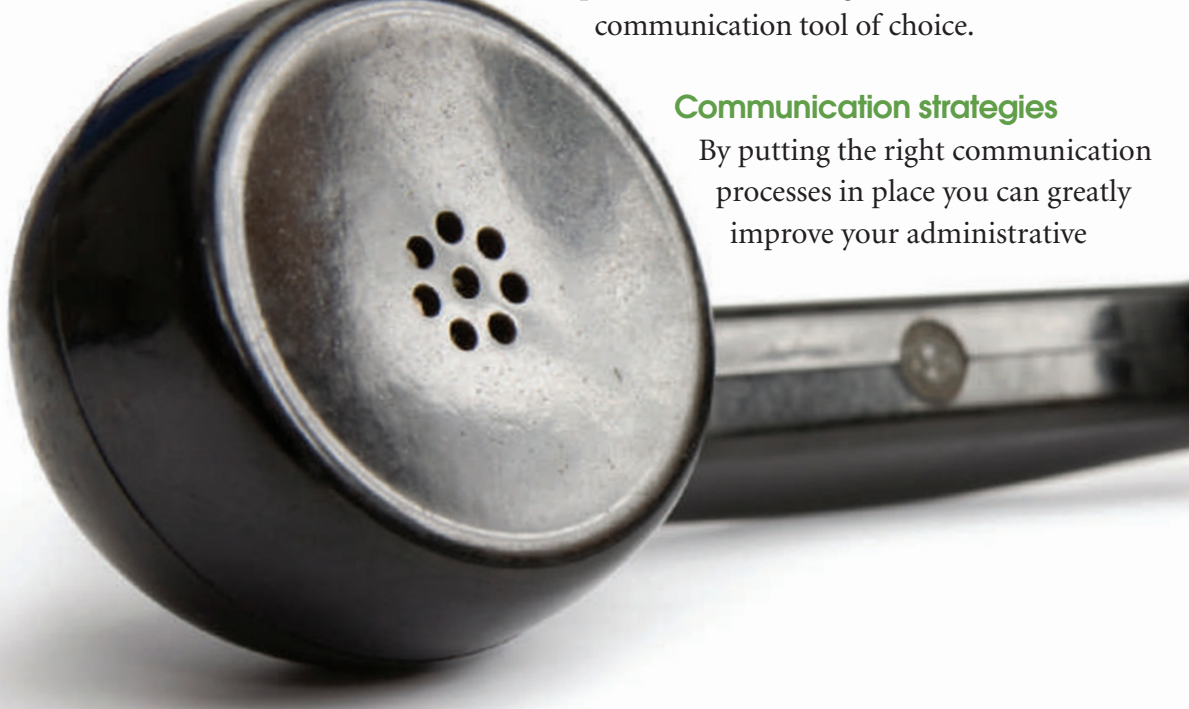

efficiency, your practice's ability to retain its existing customer base, and your practice's ability to win new business. Your direct communication with your patients largely falls into two categories: incoming (or disruptive) and outgoing (or proactive).

Dental practices are used to people phoning and emailing throughout the day to book appointments or ask questions (see Figs 1-2). This is a disruptive process but cannot be avoided completely. What you can do is minimise the disruption by making sure staff have the right tools and knowledge to deal with these incoming enquiries efficiently. The person answering the phone should be able to deal with as many of your incoming enquiries as possible without having to interrupt any other member of staff.

What dental practices are often less used to doing is proactively picking up the phone and calling patients themselves. There are a number of reasons why taking a more proactive approach to your outgoing communications is important, but the principal reason among them is that it puts you in control of your relationship with your patient. 


\section{Turning online enquiries into patients}

Whether you are communicating with a potential patient or an existing patient, your goal is to build a relationship with them. The single best way to do this is to speak with the patient as soon as you possibly can. This is particularly important with regard to online enquiries.

As the number of people who contact your dental practice by email grows over time, more of your resources are going to be taken up by dealing with them. Your goal here should be to move the conversation offline as soon as possible. Speaking to the patient directly builds far more trust than replying by email does.

Taking the conversation offline means making outgoing calls, which even hardened telesales professionals don't particularly enjoy, but the results speak for themselves. Dental practices that only send an automatic email reply to potential patients can expect a conversion rate of less than $5 \%$. Practices that send a personalised response and also phone the patient can expect a conversion rate of $20 \%$ or more (Fig. 3 ).

\section{Exceeding expectations}

Over $40 \%$ of online enquiries are generated outside of office hours (Fig. 2), and each of these patients has a different set of expectations about how and when they will hear back from you. A few of them will give you explicit instructions, perhaps to call at a certain time or maybe to only email them. In these cases it is important that you follow their instructions to at least meet their expectations.

As a rule of thumb all other patients are waiting to hear from you as soon as possible. Your dental team needs to be aware of this and learn to deal with all overnight email and phone messages first thing in the morning. The goal here should be to not only meet the patient's expectation for a timely response, but to exceed their expectations with your professionalism.

\section{Maximising business}

One of the biggest advantages to making a phone call rather than just emailing or posting a letter is that you can be sure that the patient has received the information. It gives you complete control over your calendar of bookings and better still it gives you confidence that patients will turn up.

A patient is also much more likely to tell you that they can't make an appointment if you speak to them, giving you the chance to fill what would otherwise have been an empty slot, and accommodate the patient with a new appointment at a time convenient to them.

Remember that repeat business is just as important as new business, and existing

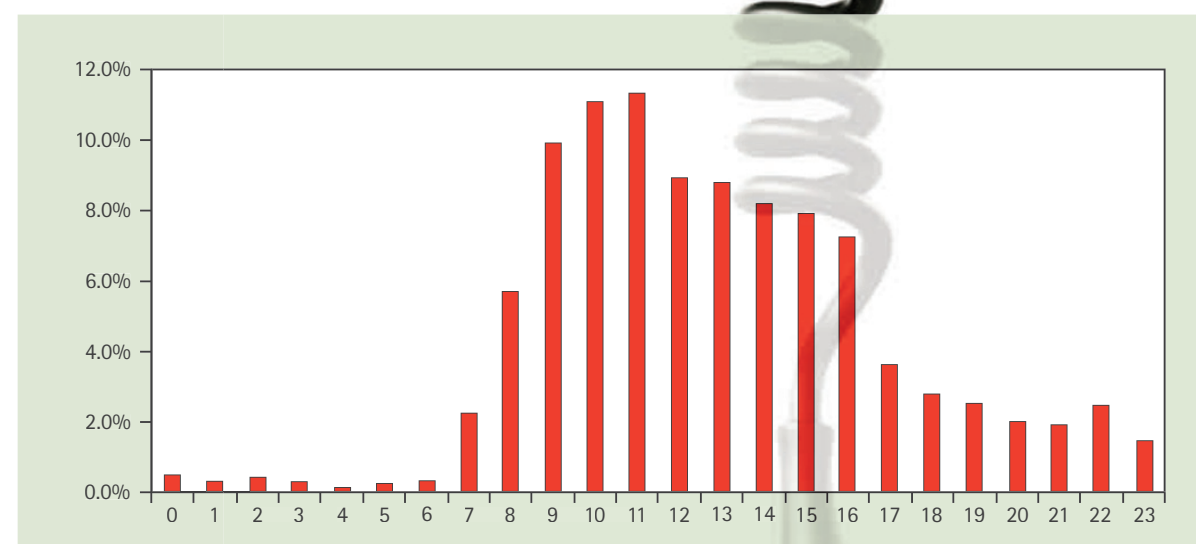

Fig. 1 Time of phone enquiries (\%) (Source: RevaHealth.com April 2010)

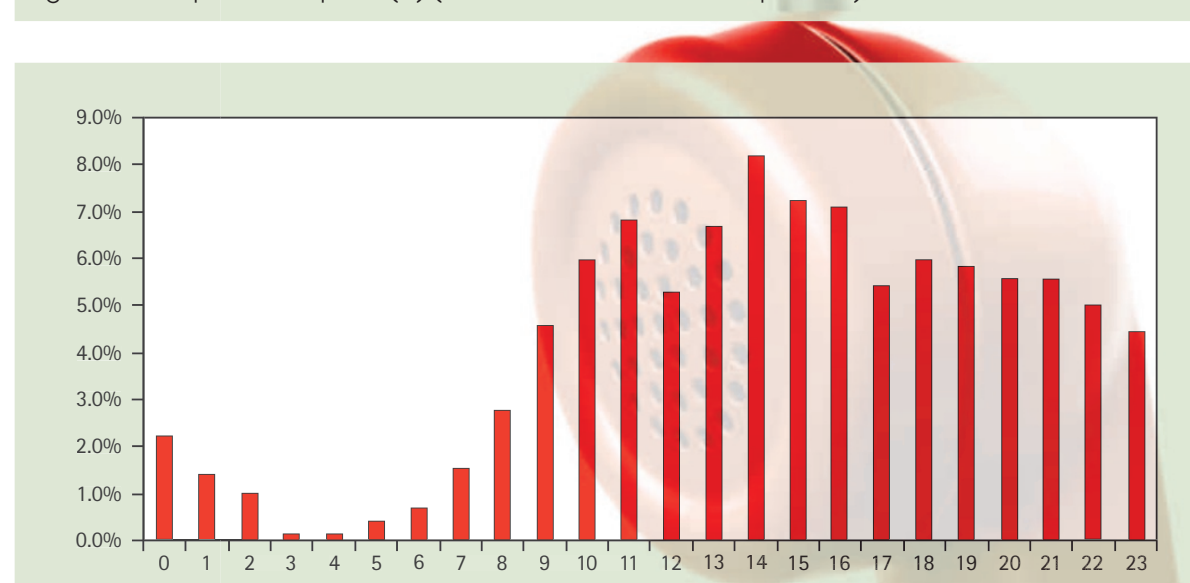

Fig. 2 Time of email enquiries (\%) (Source: RevaHealth.com April 2010)

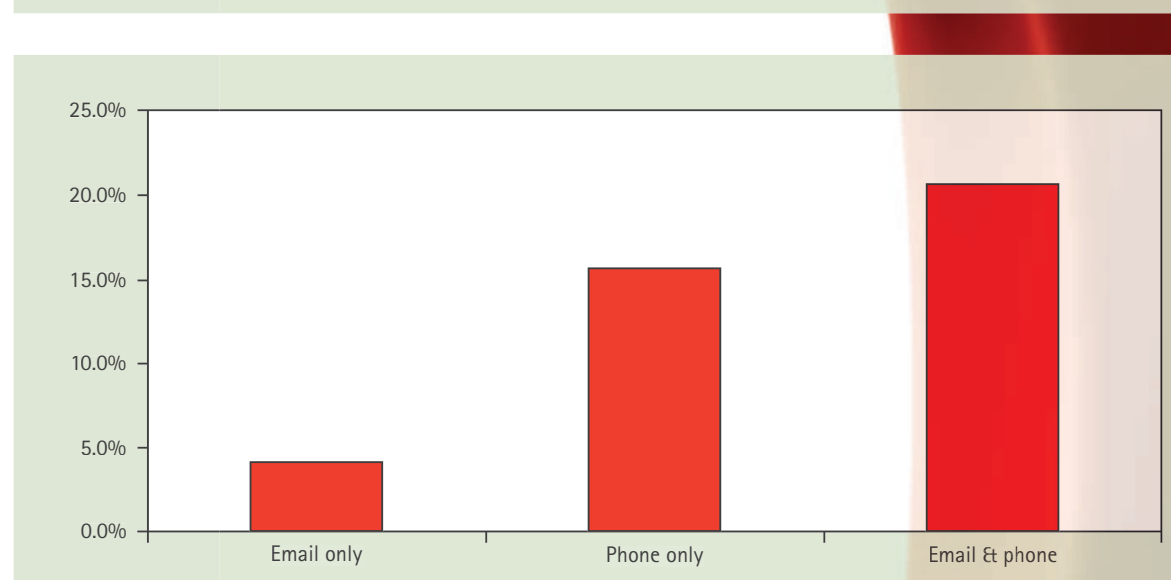

Fig. 3 Enquiry conversion rate by communication method (Source: RevaHealth.com April 2010)

customers need to have their expectations met and exceeded too. If you can manage to do this you not only retain them as a customer, you might even turn them into a fan: someone who will recommend your services publicly to their friends, family and colleagues.

\section{The tool of choice}

Whether you are trying to get a potential patient to make a booking, reminding an existing patient about an appointment, or informing patients about a new service your dental practice offers, at every stage of your relationship with your patient the phone should be your outgoing communication tool of choice. 